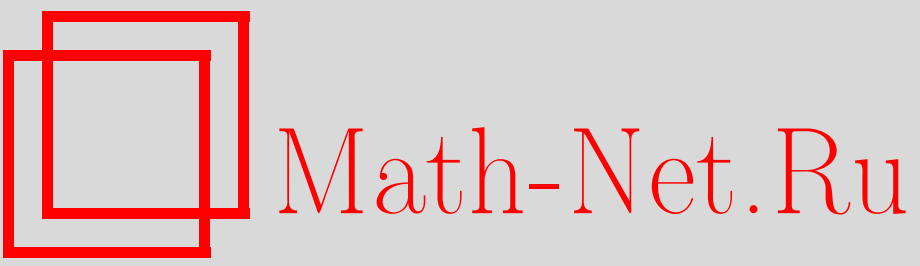

А. М. Райгородский, О нижних оценках для чисел Борсука и Хадвигера, УМH, 2004, том 59, выпуск 3, 177-178

DOI: https://doi.org/10.4213/rm752

Использование Общероссийского математического портала Math-Net.Ru подразумевает, что вы прочитали и согласны с пользовательским соглашением

http://www.mathnet.ru/rus/agreement

Параметры загрузки:

IP : 54.80 .73 .141

26 апреля 2023 г., 04:54:15 


\title{
О НИЖНИХ ОЦЕНКАХ ДЛЯ ЧИСЕЛ БОРСУКА И ХАДВИГЕРА
}

\author{
А. М. РАЙГОРОДСКИЙ
}

1. Введение и формулировка результата. Настоящая работа посвящена исследованию двух классических задач комбинаторной геометрии. Первая задача была сформулирована в 1933 г. К. Борсуком (см. [1]), и состоит она в отыскании минимального числа $f(d)$ такого, что всякое ограниченное $d$-мерное множество ненулевого диаметра может быть разбито на $f(d)$ частей меньшего диаметра. Вторая задача возникла в сороковые годы двадщатого века, благодаря работам Г. Хадвигера и П. Эрдёша, в которых впервые было определено понятие хроматического числа евклидова пространства, т.е. величины $\chi\left(\mathbb{R}^{n}\right)$, равной наименьшему количеству цветов, необходимых для раскраски $\mathbb{R}^{n}$, обладающей тем свойством, что в рамках нее точки одного цвета никогда не отстоят друг от друга на расстояние 1 (см. [2]). Отметим, что размерность в разных задачах мы обозначаем по-разному исключительно для удобства дальнейшего изложения.

Про величины $f(d)$ и $\chi\left(\mathbb{R}^{n}\right)$, которые мы также назьваем числами Борсука и Хадвигера соответственно, известно очень много. Например, детальный обзор имеющихся результатов можно найти в [3]. Невдаваясь в подробности истории вопроса, мы здесь заметим лишь, что долгое время неулучшенными оставались оценки $f(d) \geqslant(1.203 \ldots+o(1))^{\sqrt{d}}\left(\right.$ см. [4]) и $\chi\left(\mathbb{R}^{n}\right) \geqslant(1.207 \ldots+$ $o(1))^{n}$ (см. [5]). Первая из этих оценок принадлежит Дж. Кану и Г. Калаи, а вторая - П. Франклу и Р. Уилсону. Однако в серии статей 1999-2001 гг. (см. [3], [6] и [7]) автором был разработан новьй подход, позволивший заменить упомянутые неравенства более сильными: $f(d) \geqslant(1.2255 \ldots+$ $o(1))^{\sqrt{d}}, \chi\left(\mathbb{R}^{n}\right) \geqslant(1.239 \ldots+o(1))^{n}$. Дальнейшие продвижения представляются весьма проблематичными, и тем не менее совсем недавно автором был предложен метод, с помощью которого удается черезвычайно эффективно отыскивать нетривиальные нижние оценки для хроматических чисел конечных геометрических графов (см. [8]); несколько неожиданным и технически довольно тонким следствием этого метода явилась теорема, частный случай которой содержится в следующей "альтернативе":

АльтЕРнАТивА. Либо $\chi\left(\mathbb{R}^{n}\right) \geqslant(1.243 \ldots+o(1))^{n}$, либо $f(d) \geqslant(1.2305 \ldots+o(1))^{\sqrt{d}}$.

Приведенную алштернативу можно сформулировать и в виде абсолютного утверждения. Для этого достаточно определить функции $\beta=\beta(d)$ и $\kappa=\kappa(n)$ соотношениями $f(d)=(\beta+o(1))^{\sqrt{d}}$, $\chi\left(\mathbb{R}^{n}\right)=(\kappa+o(1))^{n}$. (Заметим, что, на самом деле, $\kappa$ наверняка постоянна, так как $\chi\left(\mathbb{R}^{n}\right) \leqslant$ $(3+o(1))^{n}$, см. [9]. В то же время про $\beta$ ничего подобного сказать нельзя: известно только, что $f(d) \leqslant(1.224 \ldots+o(1))^{d}$, см. [10] и [11].) В новых терминах алштернатива фактически означает, что, например, для $\beta+\kappa$ или для $\beta \kappa$ есть лучшая нижняя оценка, чем та, что вытекает из прежних работ автора [6] и [7]. С одной стороны, это обстоятельство лишний раз подверждает удивительную близость и глубокую взаимосвязанность задач (ср. [3]). С другой стороны, понятно, конечно, что формулировка альтернативы допускает значительное уточнение за счет надлежащей оптимизации в выборе необходимых для доказательства параметров. Само доказательство технически немного громоздко, и мы ограничимся здесь лишь его схематичным изложением.

2. Схема доказательства альтернативы. 1 . Пусть $n \in \mathbb{N}$ достаточно велико. Положим $a=[0.435 n], b=[0.108 n]$. Определим $p$ как минимальное простое число, болшшее, чем $\left[\frac{a+2 b}{3}\right] \sim$ $0.217 n$, так что, в силу известных теорем аналитической теории чисел, $p \sim 0.217 n$. Рассмотрим семейство векторов

$$
\begin{aligned}
\Sigma_{1}=\{\mathbf{x}= & \left(x_{1}, \ldots, x_{n}\right): x_{i} \in\{0,1,-1\}, i=1, \ldots, n \\
& \left.\operatorname{card}\left\{i: x_{i}= \pm 1\right\}=a ; \operatorname{card}\left\{i: x_{i}=-1\right\}=b\right\} .
\end{aligned}
$$

Работа выполнена при финансовой поддержке Российского фонда фундаментальных исследований (грант № 02-01-00912) и гранта Президента РФ для поддержки ведущих научных школ НШ-136.2003.1. 
Положим $M_{1}=\operatorname{card} \Sigma_{1}=C_{n}^{a} C_{a}^{b}=(2.5306 \ldots+o(1))^{n}$.

2. Предположим, что, каково бы ни было подмножество векторов $Q \subset \Sigma_{1}$, обладающее тем свойством, что скалярное произведение любых двух его элементов не равно $a-p \sim 0.218 n$, вьполнено неравенство $\operatorname{card} Q \leqslant D_{1}=(2.0359)^{n}$. Тогда $\Sigma_{1}$ образует $\left(M_{1}, D_{1}\right)$-критическую конфигурацию, отвечающую запрещенному расстоянию $\sqrt{2 p}$ (см. [3]), и, стало быть, $\chi\left(\mathbb{R}^{n}\right) \geqslant$ $M_{1} / D_{1}=(1.243 \ldots+o(1))^{n}$.

3. Допустим, предположение п. 2 неверно. Значит, найдется подмножество векторов $\Sigma_{2} \subset \Sigma_{1}$, удовлетворяющее условиям: $(\mathbf{x}, \mathbf{y}) \neq a-p$ для любых $\mathbf{x}, \mathbf{y} \in \Sigma_{2}$ и $M_{2}=\operatorname{card} \Sigma_{2}>D_{1}$. Учитывая эти условия, с помощью линейно-алгебраического метода в комбинаторике можно доказать следующую лемму.

Лемма. Если семейство векторов $Q \subset \Sigma_{2}$ таково, что $(\mathbf{x}, \mathbf{y}) \neq a-2 p \sim 0.001 n$ для любьх $\mathbf{x}, \mathbf{y} \in Q$, mо $\operatorname{card} Q \leqslant D_{2}=P(n) \max _{z} C_{n}^{z} C_{n-z}^{p-2 z}=(1.758 \ldots+o(1))^{n}(P(n)-$ некоторый полином).

4. От $n$-мерного множества векторов $\Sigma_{2}$ перейдем к $d$-мерному множеству векторов $\Sigma_{2}^{*}$ по следующему правилу: каждому $\mathbf{x}=\left(x_{1}, \ldots, x_{n}\right) \in \Sigma_{2}$ мы сопоставим $\mathbf{x} * \mathbf{x}=\left(y_{i j}\right)=$ $\left(\left(x_{i}-\delta / n\right)\left(x_{j}-\delta / n\right)\right)$, где величины $i, j$ независимо меняются в пределах от 1 до $n$, а порядок координат $y_{i j}$, разумеется, фиксирован (так что, во всяком случае, размерность $d$ аффиинного подпространства, в котором лежит $\Sigma_{2}^{*}$, не превосходит $\left.\frac{n(n+1)}{2} \sim \frac{n^{2}}{2}\right)$; при этом $\delta$ подбирается из условия $(\mathbf{x} * \mathbf{x}, \mathbf{y} * \mathbf{y})=((\mathbf{x}, \mathbf{y})-(a-2 p))^{2}$, которое должно иметь место для всех $\mathbf{x}, \mathbf{y} \in \Sigma_{2}$. Возможность осуществить последний выбор, вообще говоря, не очевидна, и в данном случае она базируется исключительно на малости величины $(a-2 p) / n \sim 0.001$, а также на фиксированности суммы $x_{1}+\cdots+x_{n}$.

Теперь нетрудно заметить, что максимальное расстояние между векторами из $\Sigma_{2}^{*}$ достигается тогда и только тогда, когда скалярное произведение их прообразов в $\Sigma_{2}$ равно $a-2 p$. Из этого факта и из леммы вытекает, наконец, оценка $f(d) \geqslant \frac{\operatorname{card} \Sigma_{2}^{*}}{D_{2}}=\frac{M_{2}}{D_{2}}>\frac{D_{1}}{D_{2}}=(1.15806 \ldots+$ $o(1))^{n} \geqslant(1.2305 \ldots+o(1))^{\sqrt{d}}$.

Альтернатива доказана.

ЗАмЕчАниЕ. В действительности альтернатива допускает некоторое усиление не только за счет оптимизации параметров типа $a, b$ и $p$, но еще и за счет привлечения дополнительных средств, связанных с задачами типа Эрдёша-Ко-Радо в комбинаторике. Именно эти средства, помимо уже описанных в данном пункте, лежат в основе метода, упомянутого перед формулировкой нашего утверждения. Желая проиллюстрировать этот метод, мы намеренно не вдавались в его детали. Однако дальнейшая разработка техники, по крайней мере, позволяет заменить обе константы 1.243 и 1.2305 числом 1.25 .

\section{СПИСОК ЛИТЕРАТУРЫ}

[1] K. Borsuk // Fund. Math. 1933. V. 20. P. 177-190. [2] H. Hadwiger // Portugal. Math. 1944. V. 4. Р. 140-144. [3] А. М. Райгородский // УМН. 2001. Т. 56. № 1. С. 107-146. [4] J. Kahn, G. Kalai // Amer. Math. Soc. (N.S.). 1993. V. 29. №1. P. 60-62. [5] P. Frankl, R. M. Wilson // Combinatorica. 1981. V. 1. №4. Р. 357-368. [6] А. М. Райгородский // УМН. 1999. Т. 54. № 2. С. 185-186. [7] А. М. Райгородский // УМН. 2000. Т. 55. № 2. С. 147-148. [8] А. М. Райгородский // Докл. РАН. 2003. Т. 392. № 3. С. 313-317. [9] D. G. Larman, C. A. Rogers // Mathematika. 1972. V. 19. P. 1-24. [10] O. Schramm // Mathematika. 1988. V. 35. № 2. P. 180-189. [11] J. Bourgain, J. Lindenstrauss // Lecture Notes in Math. 1991. V. 1469. P. 138-144.

Принято редколлегией 01.04 .2004 